\title{
High Blood Oxygen Saturation
}

National Cancer Institute

\section{Source}

National Cancer Institute. High Blood Oxygen Saturation. NCl Thesaurus. Code C50584.

A high level of the deg ree to which oxygen is bound to hemoglobin given as a percentage calculated by dividing the maximum oxygen capacity into the actual oxygen content and multiplying by 100 . Oxygen saturation usually is measured using pulse oximetry. 